\section{A147 MODULATION OF HUMORAL IMMUNE RESPONSES AS A NOVEL MECHANISM OF ACTION OF TUMOUR NECROSIS FACTOR BLOCKADE}

S Brouard, ${ }^{1}$ G Franco Salinas, ${ }^{2}$ V Jovanonic, ${ }^{1}$ F Moizant, ${ }^{1}$ P-P Tak, ${ }^{2}$ J-P Soulillou, ${ }^{1}$ D Baeten ${ }^{1,2}{ }^{1}$ INSERM U643, Nantes, France; ${ }^{2}$ Clinical Immunology and Rheumatology, Academic Medical Center/University of Amsterdam, The Netherlands

\subsection{6/ard.2010.129643x}

Objectives Based on our observations of IgM but not IgG antidsDNA antibody induction in patients treated with tumour necrosis factor (TNF) blockers, we proposed the hypothesis that TNF blockade interferes with the induction of T cell-dependent humoral responses. This study aimed to assess this hypothesis by assessing the induction of alloantibodies in a rat cardiac allograft model.

Materials and Methods LEW.1W hearts were ectopically transplanted in LEW.1A rats which were treated with anti-rat TNF or control antibody (3G8). Graft rejection was monitored clinically and serum was obtained every 5 days until day 25 . Transplanted hearts were obtained 5 days after transplantation and were assessed by histology, immunohistochemistry and quantitative RT-PCR for cytokines (Th1/Th2), TLRs and regulatory molecules.

Results ELISA analysis of serum in the LEW.1W to LEW.1A rat allotransplantation model showed a clear induction of alloantibodies in the control-treated rats from day 5 . This induction was significantly impaired by single anti-TNF treatment and completely blocked by triple anti-TNF injection. Accordingly, IgG deposition in the grafts at day 5 was significantly lower in anti-TNF $\alpha$-treated animals than in control animals ( $p=001)$, without significant differences for IgM deposition. This was histologically associated with a better conserved histological architecture (Banff grade 2-3A vs $3 \mathrm{~A}-3 \mathrm{~B})$ and a lower number of infiltrating leucocytes $(p=0.15)$ in anti-TNF-treated recipients compared to controls. In contrast with the alloantibody data, TNF blockade did not affect the Th1/Th2 balance, TLR expression and the expression of the regulatory molecules TGF $\beta$, IDO, HO-1 and FoxP3. Clinically, graft survival was prolonged from 6 days to 13 days by a single intraperitoneal injection with anti-TNF $(8 \mathrm{mg} / \mathrm{kg})$ at day 0 . Upon multiple injections (days 0,3 and 6$)$, the mean graft survival was further prolonged to 23 days. A higher dosage of anti-TNF $(15 \mathrm{mg} / \mathrm{kg})$ or concomitant treatment with suboptimal doses of ciclosporin did not have a significant additional effect.

Conclusions TNF blockade completely blocks the induction of alloantibodies, which results in a significant prolongation of graft survival in this allotransplantation model. TNF blockade may have a similar effect on humoral responses in other situations, including clinical treatment of patients with immunemediated inflammatory disorders. 